\title{
Investigation of Naturally Acquired Antibody to Haemophilus influenzae Type b Polysaccharide in China
}

\author{
Yanan $\mathrm{Li}^{\mathrm{a}, 1}{ }^{1}$, Hong $\mathrm{Li}^{\mathrm{a}, 1}$, Xiumei $\mathrm{Li}^{\mathrm{b}, 1, *}$, Pengfei Hea ${ }^{\mathrm{a}}$, Maoguang $\mathrm{Li}^{\mathrm{a}}$, Jing Tang ${ }^{a}$, Gang Shi ${ }^{a}$, \\ Cuiping Chen ${ }^{a}$, Qiang Ye ${ }^{a, *}$ \\ ${ }^{a}$ National Institute for Food and Drug Control, Tiantan Xili No.2, Beijing 100050, China \\ ${ }^{b}$ Feed Research Institute, Chinese Academy of Agricultural Sciences, No.12 Zhongguancun South St., Beijing 100081, China \\ ${ }^{1}$ The first three authors contributed equally to the project.
}

\section{Dear Sir,}

Haemophilus influenzae type b (Hib), a bacteria pathogen, is commonly found in the oropharynx of healthy unimmunized individuals [1]. Infections due to Hib are a major cause of bacterial pneumonia, meningitis, epiglottitis and septicaemia in young children throughout the world. Hib is estimated to cause at least 3 million cases of serious disease every year as well as approximately 386,000 deaths occurred in children < 5 years of age worldwide [2], It has become a major global public health problem. Hib conjugate vaccine, which has been widely used in China for more than 10 years. J Microbiol Infect Dis 2016;6(4): 197-198

In order to study the natural antibody levels among children, 13,799 serum samples of healthy children for 2 months to 5 years were collected from 15 districts (Liuzhou, Wuzhou, Longsheng, Pingle, Yangshuo, Lingchuan, Lipu, Longan, Binyang, Binhai, Xiangshui, Dafeng, Lianshui, Wuyang, Xiping) in China who had not been inoculated with Hib conjugate vaccine from 2007 to 2014. The anti-Hib IgG antibody (anti-PRP) level was measured with ELISA [3], in an attempt to provide evidence for the immune programming of Hib conjugate vaccine in China.

Out of the total 15 districts samples, the geometric mean concentrations (GMCs) and positive rate of 2 months to 5 years old children generally rising year by year in different regions, but children 2 to 11 months of age is still susceptible of Hib. So, the Hib conjugate vaccine inoculation against the focus of the crowd is needed. In addition, antibody levels were significantly different in different regions. There were no significant differences between the different genders. The antibody levels were variation with time in different age groups. In 2007, the GMCs and positive rate were not significantly different among the different age groups in any region. However, in 2007, Wu et al on the positive rate of antibody in children in some regions of Jiangsu province, the positive rate of children 1-6 months and $7-12$ months of age was $43.24 \%$ and $51.0 \%$, respectively [4]. In a similar study conducted in Turkey in 2004, the positivity rate of children 6-60 months of age was $65.35 \%$ [5]. The positive rate of children 6-23 months of age was $10-23 \%$ in Mali [6] In some literature reports, the positive rate of children aged 1-12 months was higher than in the present survey, which might have been related to the differences in age grouping and laboratory method, but the positive rate of children 1-5 years of age was lower than in the present survey, indicating that the positive rate has a more rapid rise for children in this age group in China; from 2008 to 2010, the antibody level was the highest for children 1-5 years of age and the lowest for children 6-11 months of age; the antibody level was the lowest for infants 2-5 months of age from 2011 to 2014.

In a word, although this investigation having relatively less scope, however, for public health policy makers we recommend a range which may lead to decrease the spread of Hib infection.

Correspondence: Xiumei Li, Feed Research Institute, Chinese Academy of Agricultural Sciences, No.12 Zhongguancun South St., Beijing 100081, China Email: lixiumei@caas.cn

Qiang Ye, National Institute for Food and Drug Control, Tiantan Xili No.2, Beijing 100050, China Email: qiangyee@nicpbp.org.cn Received: 22 May 2016, Accepted: 09 August 2016

Copyright (C) Journal of Microbiology and Infectious Diseases 2016, All rights reserved 
Declaration of Conflicting Interests: The authors declare that they have no conflict of interest.

Financial Disclosure: No financial support was received.

\section{REFERENCES}

1.Watt JP, Wolfson LJ, O'Brien KL, et al. Hib and Pneumococcal Global Burden of Disease Study Team. Burden of disease caused by Haemophilus influenzae type $\mathrm{b}$ in children younger than 5 years: global estimates. Lancet 2009; 374 (9693): 903-911.

2. WHO. WHO Position paper on Haemophilus influenzae type b conjugate vaccines. WER 2006;81(47): 445-452.
3. Antohyn BF, Concepcion NF, McGeary SA, et al. Immunospecificity and quantitation of an enzyme-linked immunosorbent assay for group B streptococcal antibody. J Clin Microbio 1982; I16(2): 350-354.

6. Wu T, Tao H, LI H, et al. Natural Immunity Antibody Level to Haemophilus Influenzae Type b Polysaccharide in Jiangsu Province. Nanjing Medical University 2007; 13(3): 267-269.

7. Ocaktan E, Ozyurda F, Akar N. Natural immunity to Haemophilus influenzae type Bin children of Ankara, Turkey. Pediatr Int 2004; 46(3): 280 -284.

8. Hutter J, Pasetti MF, Sanogo D, et al. Naturally Acquired and Conjugate Vaccine-Induced Antibody to Haemophilus influenzae Type b (Hib) Polysaccharide in Malian Children: Serological Assessment of the Hib Immunization Program in Mali. Am J Trop Med Hyg 2012; 86(6): 1026-1031. 